\title{
An Exploration of Knowledge and Themes on Diabetes during Outpatient Consultation in a Tertiary Referral Hospital*
}

\author{
Angelique Bea Uy, ${ }_{1}$ Harold Henrison Chiu, ${ }^{1}$ Ramon Larrazabal Jr., ${ }^{2}$ Cecilia Jimeno ${ }^{1}$ \\ ${ }^{1}$ Division of Endocrinology, Diabetes and Metabolism, Department of Medicine, \\ University of the Philippines College of Medicine-Philippine General Hospital \\ ${ }^{2}$ Department of Medicine, University of the Philippines College of Medicine-Philippine General Hospital
}

\begin{abstract}
Objectives. Effective communication has been correlated with improved outcomes in diabetes mellitus. Patient comprehension bears an effect on understanding, improving healthcare access and utilization, interaction with healthcare providers, caring for one's own health, and shared decision making. Currently, there is a gamut of information on diabetesrelated terms from various sources. However, no material has yet been available for clinical use in the third world setting. Hence, we explored the most common themes discussed during an outpatient diabetes consult in our hospital.
\end{abstract}

Methodology. Consultation audio recordings $(\mathrm{N}=96)$ and focus group discussions $(\mathrm{N}=32)$ were conducted among adults with diabetes. Transcribed results underwent qualitative content and thematic analyses to develop the conceptual framework.

Results. The study generated the following themes: diabetes mellitus diagnosis, lifestyle modification, treatment targets, hypoglycemia precautions, diabetes complications, and medication safety. There was a good understanding of these themes among patients with a higher educational attainment, however, among those with lower educational attainment, the attitude of patients toward diabetes care is paternalistic.

Conclusion. The themes discussed in outpatient diabetes consult reflects the dimensions of diabetes care mainly influenced by socio-cultural factors, patient-doctor relationships and adaptability to limitations of resources. The results will be used to develop and validate a culturally appropriate diabetes health literacy tool.

Key words: diabetes mellitus, focused group discussion, health literacy

\section{BACKGROUND}

Diabetes mellitus is recognized as a challenge for many healthcare systems. It is a chronic disabling disorder which poses a burden not only for individuals with the disease but also to society. The deterioration of glucose control increases the incidence of diabetes-related macro- and micro-vascular complications such as blindness, stroke, cardiovascular events, chronic kidney disease and lower extremity amputations. ${ }^{1}$

Despite the advancement of scientific knowledge on the pathology and development of novel treatments in diabetes, the burden of the disease continues to escalate. A multidisciplinary approach is key to tackle the difficulties of chronic disease management to encourage effective self-care including diet and medication adherence, promoting physical activity and participation in preventive care strategies. ${ }^{2-3}$

ISSN 0857-1074 (Print) | eISSN 2308-118x (Online)

Printed in the Philippines

Copyright (C) 2021 by Uy et al.

Received: January 9, 2021. Accepted: March 5, 2021.

Published online first: April 18, 2021.

https://doi.org/10.15605/jafes.036.01.05
Various factors have been identified as social determinants of health. Although there are many factors affecting the process of care and achieving improved health outcomes and quality of life in diabetes, provider linguistic and cultural competency has been seen as a knowledge gap needing to be addressed in culturally-specific diabetes management. Language barriers can impact the perception patients have of healthcare facilities. Simple advice ranging from what is the disease, the extent of the disease/ complications, lifestyle modifications and medication adherence might be a problem if mistranslated.

Addressing language barriers caused by medical terminology may potentially curb health inequities. ${ }^{4}$ Patient understanding is crucial to maximize participation in their own management to achieve adequate care and improve disease status; on the other side of the equation are the quality and capacities of the healthcare systems, organizations and professionals rendering care. ${ }^{5-6}$ Health

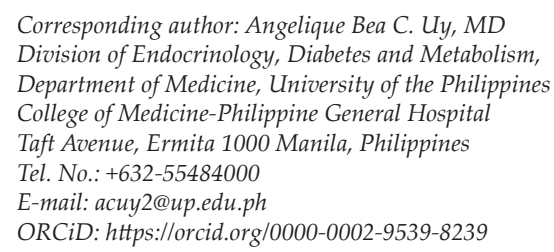

\footnotetext{
* This paper won $1^{\text {st }}$ place in the PCP Most Outstanding Researches 2021, 51 $1^{\text {st }}$ Philippine College of Physicians Annual Convention under the descriptive category, May 2 - 5, 2021; the paper was also presented as E-poster and awarded with an International Travel Grant in the 17th Asia Oceania Congress of Endocrinology and $8^{\text {th }}$ Seoul International Conference of Endocrinology and Metabolism, Virtual Congress, October 28 - 31, 2020.
} 
professionals must establish and understand a patient's health literacy before delivering interventions or education. ${ }^{7}$

The Institute of Medicine describes an attribute of a health literate organization as having healthcare providers who use health literacy strategies during interpersonal communications with patients. ${ }^{8}$ One significant challenge in the use of medical terminologies in health consults is the mismatch in functional and communicative aspect of the health literacy domain between the patient and the healthcare provider. A recent systematic review of health literacy sensitive diabetes interventions showed significant improvements in diabetes control with use of plain language, limiting teaching to 3 to 5 key points, and incorporating teach-back to ensure comprehension. However, healthcare providers have not consistently adopted these techniques. ${ }^{9-11}$

Currently, there is a gamut of information on diabetes terms to be found on the internet, in textbooks, handouts, etc. No material has yet been compiled based on the diabetes-related terms used in clinical practice in the third world and how these are defined in a culturallyappropriate perspective of both the healthcare provider and the patient. ${ }^{12}$

Effective communication has been correlated with improved outcomes. Patient comprehension during a medical consult has an effect on disease understanding; improving access and utilization of healthcare; interacting with healthcare providers; caring for one's own health; participating in health debates and decision making and greater satisfaction. ${ }^{13}$ Doctors frequently introduce medical terms during consults that may not necessarily be highfalutin, but due to the lack of simpler terms are still used, impairing effective communication. ${ }^{14-16}$ As such, it is important to identify commonly used diabetes medical themes during consult.

The aim of the study was to generate the most common themes discussed during an outpatient diabetes consult in a tertiary hospital, determine the level of knowledge and understanding of patients with diabetes of the most common diabetes medical terminologies utilized during consults, and to develop a conceptual framework determining the factors affecting diabetes care relating to the themes generated. This shall pave the way to better and effective communication of the previously known 'medical jargon' into scientifically meaningful terms because of better comprehension. This will lead to patient empowerment and decision-making with respect to self-care, medication adherence and complications prevention. In addition, the results of this study will also contribute to Filipino lexicography. Terminologies and definitions generated may be of use in constructing materials for more effective translation and expression of medical terms related to diabetes care.

\section{METHODOLOGY}

Our study was carried out in accordance with the principles outlined in the 2015 Declaration of Helsinki. It was approved by the Institutional Review Board and Research Ethics Board of the University of the Philippines Manila (UPMREB Code: 2018-610, RGAO Registration No.: 2018-1166). Informed consent was obtained from the patients prior to the audio recordings and focused group discussions.

\section{Study Design and Setting}

This was a qualitative study consisting of transcription of patient consult recordings and focus group discussions conducted within the premises of the outpatient department of a tertiary hospital in the Philippines.

\section{Study Procedures and Outcomes}

\section{Phase I. Diabetes Consultation Recordings and Transcription}

The sample size for the audio recordings was 96 to represent the population of the physicians who conduct consultations in patients with diabetes. The recordings were stratified according to patient age $(18-60$ years vs. more than 60 years) and educational status (high school graduate or less vs. college or more). The patients were recruited in this phase of the study using purposive sampling. Recordings were undertaken once the patient has signified their informed consent during consults at the family medicine, internal medicine and endocrinology clinics, respectively (Table 1 ).

To generate a list of medical terms used during a diabetes consultation encounter, transcriptions of consultation audio recordings (with patient consent) and content analysis were performed by an independent observer. The diabetes-related medical terms were quantitatively tallied on how many times they were mentioned during consults, taking into consideration synonymous terms and grouping together of similar concepts before the final list of terms was generated.

\section{Phase II. Focus Group Discussion (FGD)}

To validate the list generated from Phase I, FGDs involving a group of diabetic healthcare experts and another group of adult diabetic patients selected via purposive sampling were done with the principal investigator as facilitator (Table 2).

Table 1. Summary of the number of audio recordings from the outpatient clinics stratified according to age and educational status

\begin{tabular}{cccc}
\hline Clinic & Age range (years) & Educational status & Number of recordings \\
\hline \multirow{2}{*}{ Family Medicine } & $18-60$ & High school graduate or less & 18 \\
& More than 60 & College or more & 18 \\
\hline \multirow{2}{*}{ Internal Medicine } & $18-60$ & High school graduate or less & 18 \\
& More than 60 & College or more & 18 \\
\hline \multirow{2}{*}{ Endocrinology } & $18-60$ & High school graduate or less & 12 \\
& More than 60 & College or more & 12 \\
\hline
\end{tabular}




\begin{tabular}{|c|c|c|c|}
\hline Group & Age range (years) & Educational status & Number of participants \\
\hline 1 & $18-60$ & \multirow{2}{*}{ High school graduate or less } & 8 \\
\hline 2 & More than 60 & & 8 \\
\hline 3 & $18-60$ & \multirow{2}{*}{ College or more } & 8 \\
\hline 4 & More than 60 & & 8 \\
\hline
\end{tabular}

The panel experts were as follows: 3 endocrinologists, 2 internal medicine physicians and 2 family medicine physicians. We did not include paramedical staff and social scientists in our study. The groups of adult patients with diabetes comprised of eight participants each, large enough to keep the conversation going, at the same time small enough to prevent people from being left out of the discussion,; ${ }^{17}$ each group was categorized according to age and educational attainment (Table 2).

The activity was conducted in a quiet room where the participants, including the facilitator, were seated faceto-face in a circle. It commenced with the facilitator first explaining the purpose and outline of the FGD. The following discussion points were tackled: 1) Identify the factors affecting health outcome in diabetes care; 2) Identify the role of diabetes consultation advice on diabetes control; 3) Identify medical terms commonly used during the consult; 4) Identify which medical terms are easily to poorly understood; and 5) Define in their own words each diabetes medical term.

Each participant was given a chance to speak during the entire duration of the activity. The facilitator noted both verbal responses and non-verbal cues including gestures and body language. The FGD concluded with the facilitator summarizing the key points of the discussion. The team reviewed the responses in order to validate the transcribed responses from the consultation recordings and the FGD responses.

Consensus was derived by the Delphi method, the standard technique for achieving convergence of opinion from a panel of experts. The following were the points for consensus: 1) Factors affecting health outcome in diabetes care; 2) Role of diabetes consultation advice on diabetes control; 3) Medical terms commonly used during the consult; 4) Medical terms that are difficult to understand; and 5) Own definition of the medical term diabetes. The Delphi method relies on a system of iteration and feedback to achieve a summation of comments, making each panel member aware of the range of opinions and the reasons underlying them. The method is also notable for its ability to provide anonymity, reducing the effects of group pressure for conformity, the presence of a controlled feedback process, and the suitability of a variety of statistical analysis techniques to interpret the data. ${ }^{18}$

The process took place over several rounds: In the first round, all panel experts were emailed a transcribed copy of the consultation recordings and FGD outputs. They were asked to individually place their opinions, as well as the underlying reasons for these. These responses were collected and summarized by a facilitator, who kept the identity of each panel expert anonymous. The generated diabetes medical terms commonly used during consultations were sent back to the individual panel experts for review, in light of the other panel members' replies.

This second round reveals areas of agreement and disagreement, and consensus begins forming. ${ }^{19}$ Finally, during a third round (the minimum number recommended), the revised output was again sent back to the individual panel members, and the version approved by the majority $\left(50 \%+1\right.$, or at least four experts) was accepted..$^{20}$

A conceptual framework was generated using the terms from the audio recordings, focus group discussion and consensus from the panel experts in order to summarize the important key factors that affect diabetes care during outpatient consults.

\section{RESULTS}

\section{Phase I. Diabetes Consultation Recordings and Transcription}

A total of 96 diabetes consults recordings were obtained from clinics in the Philippine General Hospital Outpatient department handling patients with diabetes: family medicine (FM) $(n=36)$, internal medicine (IM) $(n=36)$, and endocrinology $(n=24)$. The patient groupings are summarized in Table 1. Diabetes consultation duration varied from 15 to 45 minutes in the FM Clinic, 2 to 49 minutes in the IM Clinic, and 3 to 35 minutes in the Endocrinology clinic.

The top ten most commonly used diabetes medical terminologies during a patient-doctor encounter in an outpatient clinic include: (1) Fasting Blood Sugar (FBS); (2) Hypoglycemia and its symptoms; (3) HbA1c; (4) Creatinine in relation to diabetic nephropathy; (5) Diet and Exercise; (6) BP Control; (7) DM Complications; (8) Symptoms of Diabetes Mellitus; (9) Treatment goals; and (10) Medication side effects. The most commonly repeated concept in each consult was diabetes complications. When re-examined, most complications were dominantly in lossframed messages versus gain-framed messages in which the latter highlights the benefits of a specific behavior or a risk factor. These terms were validated by a method of triangulation through facilitated focus group discussions.

\section{Phase II. Focus Group Discussion (FGD)}

Focus group discussions were held to validate the top most commonly used diabetes-related terms elicited from the audio recordings of consults. Elicited in the FGDs were questions on factors affecting outcome on diabetes care. Factors identified were knowledge of the disease; understanding of the disease entity; reiteration of the healthcare provider of treatment plans; how to act upon the disease and treatment targets; and a stable patient-doctor 


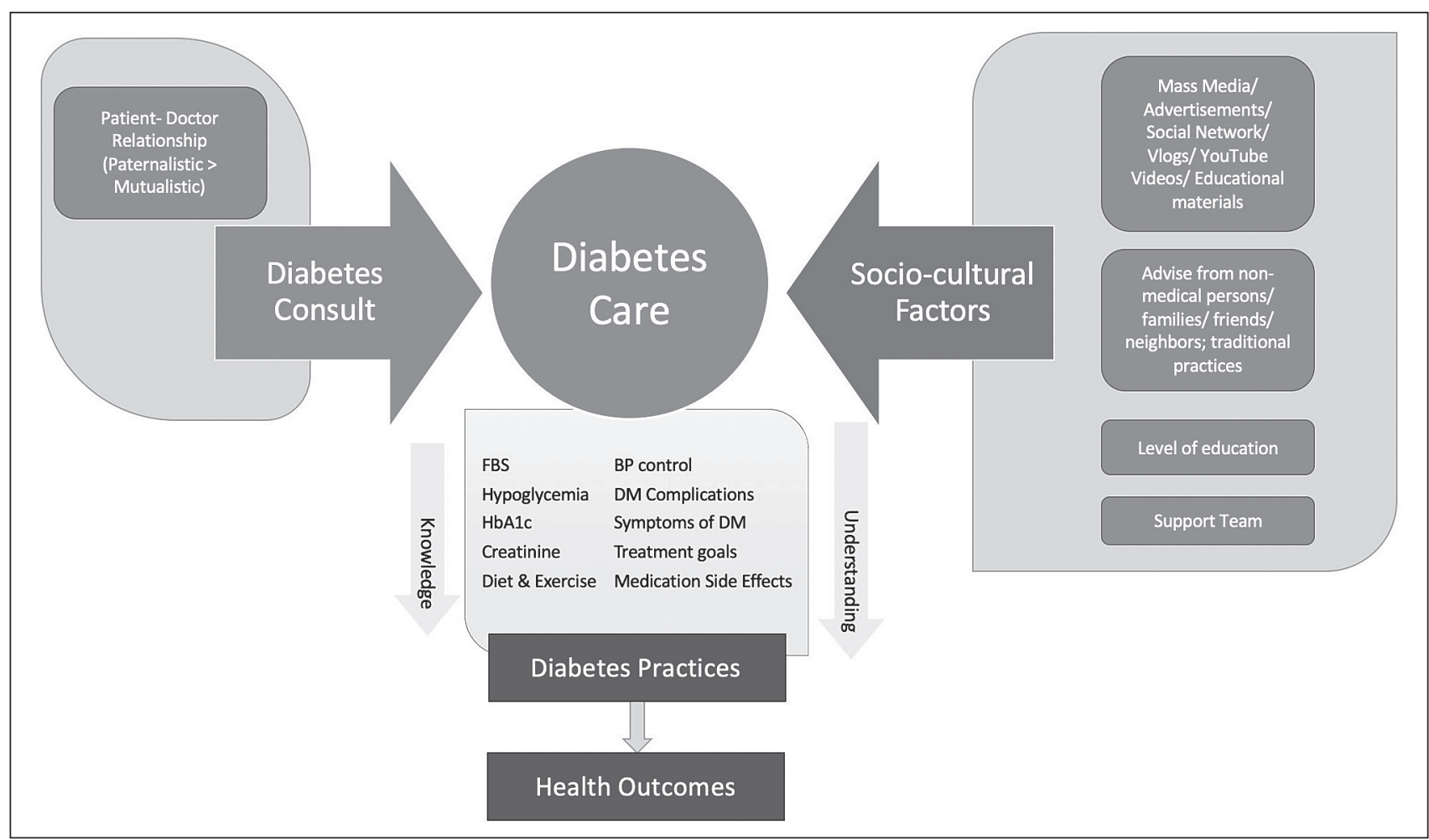

Figure 1. The conceptual framework illustrating the different identified factors that affect diabetes care during outpatient consults.

relationship guided by full trust of the doctor by the patient. It also showed a more paternalistic doctor-driven decision making versus a mutual patient-doctor relationship on the treatment plan especially on the choice of medications, the flexibility of the physician to change in treatment plans in cases of treatment failure and limitations of funds.

\section{Phase III. Generation of Conceptual Framework}

A conceptual framework (Figure 1) was then generated using the terms from the audio recordings, focus group discussion and consensus from the panel experts in order to summarize the important key factors that affect diabetes care during outpatient consults.

\section{DISCUSSION}

The Filipino language consists of a vast array of synonyms on how to express ideas. Language has the potential to benefit or hinder the attainment of proper health management. It was deemed worth further exploring each of the most common terms and themes generated during an outpatient diabetes consult to explain disease diagnosis, treatment, for patient counselling, prevention, and promote adherence to therapy.

Fasting blood sugar is a parameter used to diagnose diabetes mellitus. It is also used to monitor patients with diabetes for the appropriateness of sugar control. It is usually taken after a person has not eaten for eight to fourteen hours, usually overnight. Being the most commonly available and affordable screening and monitoring test for diabetes mellitus, it is not surprising that it is the most used diabetes-related term in a consult.

Hypoglycemia being a potentially dangerous state of low blood sugar usually less than $70 \mathrm{mg} / \mathrm{dL}$, or too little sugar in the blood presents with signs including hunger, nervousness, shakiness, perspiration, dizziness or lightheadedness, sleepiness, and confusion, is one of the symptoms most elicited by the physicians. Hypoglycemia is a diabetic emergency and a complication of treatment, and if persistent can lead to serious morbidities including but not limited to heart attacks, and even death. Patientinitiated Filipino terms pertaining to hypoglycemia includes symptoms such as nanginginig, nangangatog, labis na pagkagutom, nahihilo, lupaypay, inaantok, pagkawala ng malay. Noticeably, hypoglycemia treatment was not regularly advised in the Family Medicine and Internal Medicine clinics compared to the IM- Endocrine clinic. From the recorded consultation, around 70\% from the IMEndocrine recordings were insulin requiring, versus $40 \%$ in Family Medicine and Internal Medicine clinics. Simple culturally available measures to address hypoglycemia include consuming three pieces of hard candies, or a half-cup of juice or regular soft drinks; and to avoid skipping meals.

Hemoglobin A1c (HbA1c) is a test that measures a person's average blood sugar level over the past 2 to 3 months. Hemoglobin is the part of the red blood cell that carries oxygen to the cells and sometimes joins with the glucose in the bloodstream most especially in the setting of chronic hyperglycemia. Generally, this blood test is well understood by patients with diabetes with higher level of education i.e., some college or more, included in the study. This is their measure for control of blood sugars. It was elicited both from the individual audio recordings and the FGDs that there was a good understanding as to the purpose of this test.

Creatinine is another commonly inquired term during a diabetes consult. It is a surrogate marker for renal 
dysfunction, as it is a waste product from protein in the diet and from the muscles of the body, and removed from the body by the kidneys. This term is simply known as crea to the patients with diabetes. The association that as the creatinine increases, kidney function decreases is widely understood by our patients. Diabetes nephropathy is equated to kidney damage, kidney failure, and dialysis.

Lifestyle modifications through revision of diet and institution of exercise have also been reiterated during consults. Queries have focused primarily on the proper nutrition composition and the types of exercises best suited for patients with diabetes. The usual advice given by physicians include adapting the pinggang pinoy, a lowfat, low-calorie diet and moderate exercise usually brisk walking for at least 30 minutes, 5 days a week. Diabetes meal planning options for meal substitutions for different food groups are given to patients, however, the details of such interventions are often left to the nutritionist for further discussion. Referrals to the dietary service are done after each consult most commonly observed from the IM-Endocrine clinic.

Blood pressure, simply known as BP or presyon to patients control is emphasized in diabetes management. It has been shown that a good BP control of less than $140 / 90$ is targeted for patients with diabetes for slowing down kidney damage and minimizing albuminuria. The initiation of ACE-inhibitors or ARBs are given as options to patient to achieve this. However, this is not prioritized by patients with diabetes included in the study because of the lack of knowledge on the effects of blood pressure control on kidney function and deterioration.

Diabetes mellitus complications included in consults aside from nephropathy, are neuropathy including bilateral loss of sensation in distal extremities specifically the hands and the feet described as pamamanhid, pangangalay ng mga kamay at paa, matagal na paghilom ng sugat, pagkaputol ng paa, erectile dysfunction, constipation, delayed digestion manifesting as nausea, vomiting or bloating pagkabilis mabusog, autonomic neuropathy presenting as orthostatic dizziness pagkahilo lalo na kapag nagbago ng posisyon; retinopathy presenting as loss of vision panlalabo ng mata, pagkabulag; and macrovascular complications including heart attack, heart failure, stroke, and amputations.

Symptoms of Diabetes Mellitus elicited are polyuria, nocturia, increased thirst, unintentional weight loss, and paresthesia. These terms are translated as madalas na pagihi, labis na pagka-uhaw, pamamayat o pagbabawas ng timbang, and pamamanhid ng mga kamay at paa.

Treatment goals for patients with diabetes are inquired by patients and discussed by physicians. Different targets of HbA1c, fasting blood sugar, BP, lipid level, and weight loss are set depending on age, and the presence of co-morbidities. Generally, for younger adults with diabetes without $\mathrm{CV}$ risk an $\mathrm{A} 1 \mathrm{c}$ of $6.5-7.0 \%$ is targeted, and if older with co-morbidities, a more relaxed target is recommended. This is individualized and explained to each patient.

Medication side-effects are often a cause of worry for patients with diabetes. More so, the bulk of the information is published on social media, websites, newspapers, and advice from neighbors about unvalidated effects of some medications, i.e., metformin causing cancer or renal failure, diabetes medications causing weakness, use of insulin as a 'death sentence' in a patient's diabetes state. Physicians should additionally educate patients regarding medication side-effects and efficacy of diabetes control during consults. Furthermore, the use of non-FDA approved food supplements with no therapeutic value were also brought up. Common lay terms describing sideeffects include pananakit ng tyan, pagsusuka, labis na pagbaba ng lebel ng asukal sa dugo, pananaba.

The diabetes consultation has a key role in filling in the knowledge gap about the disease, and its consequences on the health outcomes of each patient. However, the patients are admittedly easily lured by mass mediaadvertisements/ TV commercials, YouTube videos, advice from colleagues, friends and neighbors, and social media releases by the non-experts.

Although it occurred in the top ten most commonly used terms in diabetes consults, the most poorly understood concept is that of the medication efficacy and side-effects. This serves as a limitation to achieving adherence to medication, good glycemic control, and better health outcomes.

The FGD group with a lower educational background is more or less familiar with the diabetes-related consultation terms, however, they are more reliant on its implications, and treatment adjustments to their physicians, rather than understanding the totality and course of their diabetes management. How this will affect treatment outcomes is yet to be determined in future studies.

The themes we have elicited in our study will allow us to develop a culturally-appropriate diabetes health literacy tool. The use of this tool will allow us, diabetes healthcare providers, facilitate better and more effective communication resulting in better patient comprehension with regards to self-care, medication adherence and complication prevention, and further to develop a diabetes education approach that is standardized and fit in our healthcare system. As some studies have shown, some patients do not think of a diabetes consult, including diabetes education a necessity because they do not consider themselves ill. However, we have to help patients realize that knowing the course of the disease and diabetes management can influence their health outcomes.

\section{CONCLUSION}

In summary, the themes discussed in outpatient diabetes consults reflects the dimensions of diabetes care, being influenced mainly by socio-cultural factors, patient's having a good grasp of the disease process, a trusting patient-doctor relationship and adaptability to limited resources. The study results will be used to develop and validate a culturally appropriate diabetes health literacy tool.

Statement of Authorship

All authors certified fulfillment of ICMJE authorship criteria. 


\section{Author Disclosure}

Drs. Uy, Chiu and Larrazabal declared no conflict of interest. Dr. Jimeno is the Vice Editor-in-Chief of JAFES.

\section{Funding Source}

None.

\section{References}

1. Effect of intensive blood-glucose control with metformin on complications in overweight patients with type 2 diabetes (UKPDS 34). UK Prsopective Diabetes Study (UKPDS) Group. Lancet. 1998;352(9131):854-65. PMID: 9742977.

2. Saaddine JB, Cadwell B, Gregg EW, et al. Improvements in Diabetes Processes of Care and Intermediate. Ann Intern Med. 2011;334 (Suppl 1):S89-96. PMID: 21193633. PMCID: PMC3006053. https:// doi.org/10.2337/dc11-S089. PMID: 16585660. https://doi.org/10.7326/ 0003-4819-144-7-200604040-00005.

3. Funnell MM, Brown TL, Childs BP, et al. National standards for diabetes self-management education. Diabetes Educ. 2007;33(4):599-600,6024,606. PMID: 17684162. https://doi.org/10.1177/0145721707305880.

4. Berkman ND, Sheridan SL, Donahue KE, Halpern DJ, Viera A, Crotty $\mathrm{K}$, et al. Health literacy interventions and outcomes: An updated systematic review. Evidence report/technology assessment number 199. Search. 2011;941. https://www.ahrq.gov/downloads/pub/ evidence/pdf/literacy/literacyup.pdf.

5. Ishikawa $\mathrm{H}$, Yano E. The relationship of patient participation and diabetes outcomes for patients with high vs. low health literacy. Patient Educ Couns. 2011;84(3):393-7. PMID: 21388773. https://doi. org/10.1016/j.pec.2011.01.029.

6. van der Heide I, Poureslami I, Mitic W, Shum J, Rootman I, Fitzgerald JM. Health literacy in chronic disease management: A matter of interaction. J Clin Epidemiol. 2018;102:134-8. PMID: 29793001. https://doi.org/10.1016/j.jclinepi.2018.05.010.

7. Raynor DKT. Health literacy. BMJ. 2012;344:e2188. PMID: 22442354. https://doi.org/10.1136/bmj.e2188.

8. Brach C, Keller D, Hernandez LM, et al. Ten attributes of health literate health care organizations. Participants in the workgroup on attributes of a health literate organization of the IOM Roundtable on health literacy. NAM Perspectives. National Academy of Medicine, Washington, DC; 2012. https://nam.edu/wp-content/uploads/2015/06/ BPH_Ten_HLit_Attributes.pdf.
9. Kim SH, Lee A. Health-literacy-sensitive diabetes self-management interventions: A systematic review and meta-analysis. Worldviews Evid Based Nurs. 2016;13(4):324-33. PMID: 27104337. https://doi. org/10.1111/wvn.12157.

10. Rozier RG, Horowitz AM, Podschun G. Dentist-patient communication techniques used in the United States: The results of a national survey. J Am Dent Assoc. 2011;142(5):518-30. PMID: 21531934 https://doi.org/10.14219/jada.archive.2011.0222.

11. Howe CJ, Walker D. 002-Pediatric Diabetes Educators' use of recommended health literacy communication techniques. J Pediat Nurs. 2017;34(4):101-2. https://doi.org/10.1016/j.pedn.2017.02.011.

12. Travis LB. Youth education: Diabetes dictionary. Diab Care 1978;1(5):311-3. https://doi: 10.2337/diacare.1.5.311.

13. Ley P. Communicating with patients: Improving communication satisfaction and compliance. New York, NY, US: Croom Helm: Nelson Thornes; 1988 .

14. Blackman J, Sahebjalal M. Patient understanding of frequently used cardiology terminology. Br J Cardiol. 2014;21:39. https://doi org/10.5837/bjc.2014.007.

15. Zeng QT, Tse T. Exploring and developing consumer health vocabularies. J Am Med Inform Assoc. 2006;13(1):24-9. PMID: 16221948. PMCID: PMC1380193. https://doi.org/10.1197/jamia.M1761.

16. Chapman K, Abraham C, Jenkins V, Fallowfield L. Lay understanding of terms used in cancer consultations. Psychooncology. 2003;12(6) 557-66. PMID: 12923796. https://doi.org/10.1002/pon.673.

17. Adams A, Cox AL. Questionnaires, in-depth interviews and focus groups. In: Research Methods for Human Computer Interaction. Cambridge, UK: Cambridge University Press; 2008.

18. Douglas D. A comparative study of the effectiveness of decision making processes which utilize the Delphi and leaderless group methodologies. Vol. 12, Practical Assessment Research and Evaluation. Ohio State University. Columbus: Unpublished doctoral dissertation; 1983.

19. Jacobs JM. Essential assessment criteria for physical education teacher education programs: A Delphi study. West Virginia University. Morgantown; 1996.

20. Hsu CC, Sandford BA. The Delphi technique: Making sense of the consensus. Pract Assessment, Res Eval. 2007;12(10):1-8 https://scholarworks.umass.edu/cgi/viewcontent.cgi?article=1177 \&context=pare.

Authors are required to accomplish, sign and submit scanned copies of the JAFES Author Form consisting of: (1) Authorship Certification, that authors contributed substantially to the work, that the manuscript has been read and approved by all authors, and that the requirements for authorship have been met by each author; (2) the Author Declaration, that the article represents original material that is not being considered for publication or has not been published or accepted for publication elsewhere, that the article does not infringe or violate any copyrights or intellectual property rights, and that no references have been made to predatory/suspected predatory journals; (3) the Author Contribution Disclosure, which lists the specific contributions of authors; (4) the Author Publishing Agreement which retains author copvright, grants publishing and distribution rights to JAFES, and allows JAFES to apply and enforce an Attribution-Non-Commercial Creative Commons user license; and (5) the Conversion to Visual Abstracts (*optional for original articles only) to improve dissemination to practitioners and lay readers Authors are also required to accomplish, sign, and submit the signed ICMJE form for Disclosure of Potential Conflicts of Interest. For original articles, authors are required to submit a scanned copy of the Ethics Review Approval of their research as well as registration in trial registries as appropriate. For manuscripts reporting data from studies involving animals, authors are required to submit a scanned copy of the Institutional Animal Care and Use Committee approval. For Case Reports or Series, and Images in Endocrinology, consent forms, are required for the publication of information about patients; otherwise, appropriate ethical clearance has been obtained from the institutional review board. Articles and any other material published in the JAFES represent the work of the author(s) and should not be construed to reflect the opinions of the Editors or the Publisher.

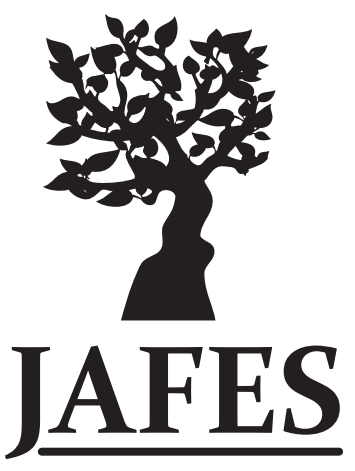

\section{Unique, interesting, enlightening. Your case report and the JAFES.}

